\title{
Manejo del aneurisma roto de aorta abdominal: un reto para el cirujano general
}

\author{
Management of ruptured abdominal aortic aneurysm: \\ a challenge for the general surgeon
}

\author{
Brandon Steven Aparicio ${ }^{1} \mathbb{D}$, Marcia Santafe-Guerrero ${ }^{1} \mathbb{D}$, Mauricio Pedraza Ciro $^{2} \mathbb{D}$, \\ Jean Pulido ${ }^{1} \mathbb{D}$, Laura Padilla ${ }^{3}$, Luis Felipe Cabrera ${ }^{4} \mathbb{D}$
}

1 Estudiante, Departamento de Medicina, Universidad El Bosque, Bogotá, D.C. Colombia.

2 Médico, residente de Cirugía general, Universidad El Bosque, Bogotá, D.C. Colombia.

3 Médico, Universidad El Bosque, Bogotá, D.C. Colombia.

4 Médico, especialista en Cirugía general, Universidad El Bosque, Bogotá, D.C. Colombia.

\section{Resumen}

Introducción. El aneurisma aórtico abdominal roto, tiene una mortalidad del 80 \% al 90 \%. Para su reparación existe una técnica abierta y otra endovascular, las cuales tienen diferencias entre sus beneficios y complicaciones. El método de elección en la actualidad para la corrección de esta alteración anatómica es endovascular, sin embargo, no es el más usado, porque no se cuenta todo el tiempo con el equipo humano de cirugía vascular para su realización.

Caso clínico. Ingresa a urgencias un paciente en estado de shock de origen desconocido, con dolor abdominal de 24 horas de evolución. Se realiza una tomografía con contraste que demuestra un aneurisma aórtico abdominal infrarrenal roto. Debido a que no se contaba con el equipo de cirugía vascular, es llevado de urgencia a una corrección abierta que duró 153 minutos, con un sangrado intraoperatorio de 1754 cc. Fue dado de alta a los 12 días postoperatorios sin ninguna complicación.

Discusión. La reparación endovascular del aneurisma aórtico abdominal roto es la mejor elección, ya que muestra mayores beneficios en comparación con la reparación abierta, sin embargo, no es el más utilizado, porque se necesita de un personal bien entrenado en cirugía endovascular, por lo que, dentro de la formación de los cirujanos generales, se tiene que incluir el aprendizaje de las técnicas abiertas.

Palabras claves: aneurisma aórtico; abdominal; ruptura; reparación; endovascular; cirugía general; cirugía vascular.

Fecha de recibido: 22/11/2019 - Fecha de aceptación: 24/02/2020 - Fecha de publicación en línea: 05/03/2021

Correspondencia: Brandon Steven Aparicio Blanco. Calle 95 \# 71-11. Bogotá, D.C. Colombia.

Correo electrónico: brandonaparicio03@gmail.com. Teléfono: 3118419650

Citar como: Aparicio BS, Santafe-Guerrero M, Pedraza Ciro M, Pulido J, Padilla L, Cabrera LF. Manejo del aneurisma roto de aorta abdominal: un reto para el cirujano general. Rev Colomb Cir. 2021;36:366-71. https://doi.org/10.30944/20117582.498

Este es un artículo de acceso abierto bajo una Licencia Creative Commons - BY-NC-ND https://creativecommons.org/licenses/by-nc$\mathrm{nd} / 4.0 /$ deed.es 


\begin{abstract}
Introduction. The ruptured abdominal aortic aneurysm has a mortality of $80 \%$ to $90 \%$. There is an open and an endovascular techniques for its repair, which have differences between their benefits and complications. The method of choice for the correction of this anatomical alteration is endovascular; however, it is not the most frequently used, mainly because the vascular surgical team is not available all the time to perform it.
\end{abstract}

Clinical case. A patient in a state of shock of unknown origin was admitted to the emergency room, with abdominal pain of 24 hours of evolution. A contrast-enhanced CT scan demonstrated a ruptured infrarenal abdominal aortic aneurysm. Due to the lack of a vascular surgery team, the patient was rushed for an open surgery that lasted 153 minutes, with an intraoperative bleeding of $1754 \mathrm{cc}$. He was discharged 12 days after surgery without any complications.

Discussion. Endovascular repair of ruptured abdominal aortic aneurysm is the best choice, since it shows greater benefits compared to open repair. However, it is not the most widely used because it requires well-trained personnel in endovascular surgery. Therefore, learning of open techniques must be included in the training of general surgeons.

Keywords: aortic aneurysm; abdominal; rupture; repair; endovascular; general surgery; vascular surgery.

\section{Introducción}

El aneurisma de aorta abdominal (AAA), se define por un aumento mayor del $50 \%$ del diámetro normal del vaso, su localización más frecuente es a nivel infrarrenal en un $80 \%$ de los casos, y cuando ocurre una ruptura, su mortalidad es del $80 \%$ al $90 \%{ }^{1}$. El manejo del AAA data del siglo XIX, sin embargo, solo fue hasta 1952 cuando Dubost, realizó la primera reparación abierta con un reemplazo de homoinjerto, posteriormente en 1991 Juan Parodi, llevó a cabo la primera reparación por vía endovascular (EVAR, endovascular aneurysm repair por sus siglas en inglés), ${ }^{1,2}$.

Se ha visto que tanto el abordaje abierto como el endovascular presentan diferencias, tales como la mortalidad a corto plazo, la necesidad de seguimiento imagenológico, la estancia hospitalaria, el sangrado, el tiempo quirúrgico, la presencia de complicaciones y la necesidad de reintervenciones ${ }^{1,2,3}$.

En la actualidad el método más usado y que ha demostrado mayores beneficios para los pacientes es el EVAR, a pesar de ello el personal que realiza este tipo de procedimiento no está disponible las 24 horas del día. ${ }^{1,4}$. De tal forma, es importante que el cirujano general esté preparado y familiarizado para afrontar este tipo de urgencias vasculares. Se presenta un caso de AAA roto, manejado por un cirujano general, realizando una revisión crítica de la literatura.

\section{Presentación de caso}

Paciente masculino de 79 años, quien consulta al servicio de urgencias del Hospital Cardiovascular de Soacha, por un cuadro clínico de 24 horas de evolución, consistente en dolor abdominal localizado en la fosa iliaca izquierda, de intensidad 9/10, irradiado a la región lumbar, no asociado a fiebre, episodios eméticos ni diarreicos. Como antecedentes de importancia, paciente hipertenso crónico y fumador de 12 paquetes/año de cigarrillo.

Posterior al ingreso, el paciente presenta un cuadro de lipotimia, con una tensión arterial de $82 / 41 \mathrm{~mm} / \mathrm{Hg}$, por lo cual se considera pasar un bolo de Lactato de Ringer de 2000 cc. Al cual el paciente responde favorablemente. Al examen físico, en la palpación abdominal se aprecia defensa voluntaria en flanco izquierdo, asociada a distensión, sin signos de irritación peritoneal. Por lo anterior se decide realizar una tomografía computarizada (TC) abdominal con contraste, la cual muestra un hematoma expansivo retroperitoneal en la zona II izquierda, secundario a AAA infrarrenal roto, asociado a un escape activo, con fuga hacia la zona II y con compromiso bilateral de las arterias iliacas (figura 1). 


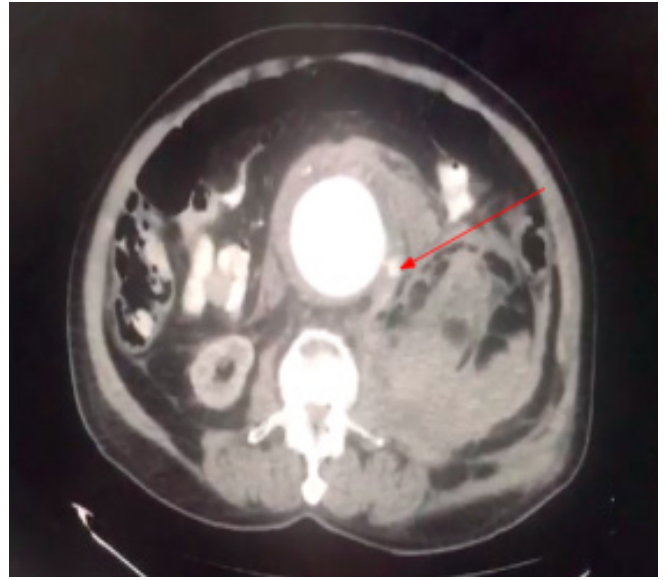

Figura 1. TC abdominal contrastada, que muestra un hematoma expansivo retroperitoneal en la zona II izquierda, secundario a AAA infrarrenal roto (flecha roja).

Al no contar con el servicio de cirugía vascular ni disponibilidad de EVAR, se lleva al paciente a salas de cirugía como una urgencia vital, para corrección del AAA roto por vía abierta. Se aborda el hematoma expansivo en la zona II retroperitoneal izquierda, a través de la fascia de Toldd, se realiza maniobra de Mattox y se identifica el cuello del AAA roto, el cual se controla con un clamp de aorta, obteniendo control vascular proximal, y se procede a colocar dos sondas Foley de 16 Fr insufladas en la luz de cada arteria iliaca, obteniendo control vascular distal. Se completa la apertura del AAA roto, se drena completamente el hematoma retroperitoneal en las zonas I y II izquierdas.

Se procedió a realizar la reconstrucción aórtica en ambas iliacas, con un injerto sintético de Dacron bifurcado de $30 \mathrm{~mm}$ con sutura vascular tipo paracaídas con polipropileno vascular 3-0 (figura 2). Por la distensión y edema severo de las asas intestinales delgadas, y para evitar un cierre a tensión de la pared abdominal, generando un alto riesgo de hipertensión intraabdominal y síndrome compartimental abdominal, se deja abdomen abierto, usando una bolsa de Borráez. Se finaliza la cirugía con un tiempo quirúrgico de 153 minutos y un sangrado de $1754 \mathrm{cc}$, y se traslada al paciente a la Unidad de Cuidados Intensivos (UCI), con soporte vasopresor a dosis mínima, para reanimación fisiológica y revisión quirúrgica. A las 48 horas se

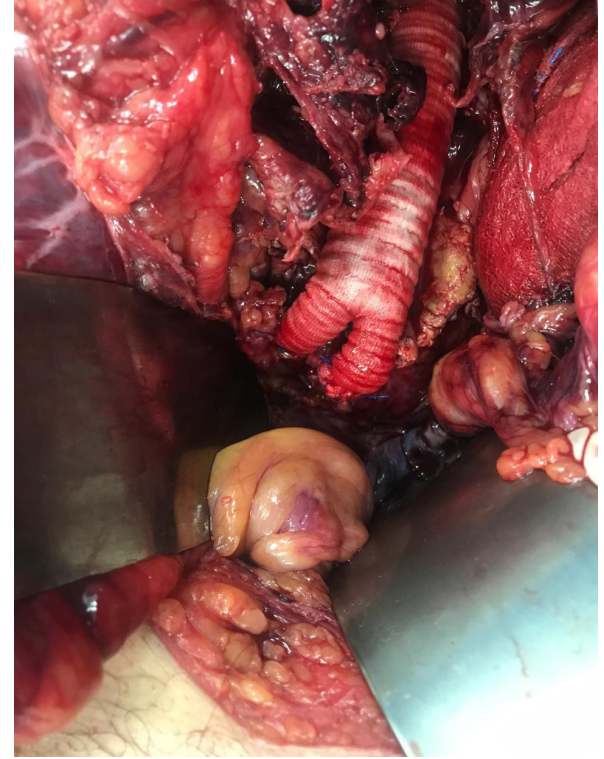

Figura 2. Reparación abierta de AAA con un injerto sintético bifurcado de $30 \mathrm{~mm}$.

reinterviene, realizando un cierre de la pared abdominal, sin nuevos hallazgos ni complicaciones. La estancia en UCI fue de seis días y la evolución clínica posterior fue favorable, siendo dado de alta a los doce días.

\section{Discusión}

La aorta abdominal tiene un diámetro de 1 a 2 $\mathrm{cm}$ en pacientes mayores de cincuenta años. Se define AAA como una dilatación mayor de $3 \mathrm{~cm}$ o un aumento mayor del $50 \%$ del diámetro normal del vaso. La degeneración, infección, inflamación y las anormalidades del tejido conectivo son las causas del aumento del calibre de esta $\operatorname{arteria}^{1}$. Su prevalencia es mayor en pacientes masculinos de raza blanca fumadores y con comorbilidades tales como la hipertensión arterial, dislipidemias, y la enfermedad pulmonar obstructiva crónica, entre otras. La ruptura del AAA depende del diámetro de la arteria. Un calibre de 4-5 cm tiene un riesgo menor del $5 \%$, cuando este es mayor de $5,5 \mathrm{~cm} \mathrm{o}$ aumenta más de $0,5 \mathrm{~cm}$ cada seis meses, alcanza un riesgo de ruptura de más del $10 \%^{1,5}$.

El hallazgo de esta anormalidad anatómica, puede aparecer de manera incidental o por un 
cuadro clínico consistente en dolor lumbar, inguinal o abdominal, masa pulsátil e hipotensión arterial, constituyendo la triada del AAA roto ${ }^{1}$. Los pacientes que presenten estos signos, deben ser llevados a estudios para descartar esta entidad y determinar la presencia de signos de ruptura. La TC contrastada es el mejor método para hacer el diagnóstico, además de ayudar en el planeamiento de la intervención si es necesaria ${ }^{1,5-7}$. Se debe realizar el manejo quirúrgico, cuando exista una dilatación de $5,5 \mathrm{~cm}$ o si hay signos de ruptura como, la salida de sangre a la cavidad abdominal o retroperitoneal ${ }^{8,9}$. En la actualidad existen dos métodos para hacer esta reparación, abierta o por vía endovascular, por lo cual se convierte en un dilema para el cirujano, ya que la elección va a depender de su experiencia, de la angulación o longitud de la arteria y de los recursos del hospital ${ }^{4-6,9}$.

La intervención quirúrgica va a ser óptima, siempre y cuando los beneficios superen los riesgos de esta ${ }^{1}$. A lo largo de las últimas décadas se han implementado diferentes técnicas, dentro de las cuales podemos encontrar la reparación abierta, que es un método invasivo y duradero que se recomienda en pacientes menores de 65 años, en quienes sus enfermedades de base no condicionen un riesgo mayor ${ }^{1,6,8,10}$. El tiempo quirúrgico en esta intervención está estandarizado en alrededor de 287 minutos, su sangrado es mayor en comparación a otras técnicas ${ }^{5,8}$. Se han visto complicaciones a corto plazo como aumento de mortalidad y de hospitalización, además de presentar a larga data obstrucciones intestinales y hernias de la pared abdominal ${ }^{3,9}$.

El EVAR se prefiere en pacientes mayores de 65 años y con presencia de comorbilidades, ya que presenta menor tiempo de sangrado, es menos invasivo y el tiempo quirúrgico es de alrededor de 241 minutos ${ }^{1,6,9}$. Presenta disminución en: estancia hospitalaria, mortalidad intraoperatoria y a los 30 días posoperatorios, en comparación con la reparación abierta, sin embargo, hay aumento de costos, riesgo de reintervenciones por complicaciones a largo plazo como endofugas, por lo cual requiere de un seguimiento estricto con imágenes, al mes, seis, y doce meses y posteriormente cada año ${ }^{1,6-9}$.
Los pacientes con AAA roto con inestabilidad hemodinámica, han sido un pilar de controversia a lo largo de los años, debido a que el EVAR en muchas investigaciones se ha excluido debido al tiempo que toma obtener los estudios para realizarla, sin embargo, se ha demostrado que no hay diferencia en cuanto a la mortalidad en AAA roto inestable versus la reparación abierta en hospitales de alto volumen, con cirujanos vasculares experimentados y todos los recursos disponibles ${ }^{9}$.

En diferentes estudios se ha demostrado los beneficios del uso de la técnica EVAR sobre la reparación abierta, como se puede demostrar en el trabajo publicado por P. del Canto Peruyera en un estudio de cohortes, entre el 2003 y 2013 en el cual se evaluaron 45 pacientes con diagnóstico de AAA roto, de los cuales, 25 fueron tratados por reparación abierta y 20 por EVAR. Encontrando que el uso del EVAR presenta una estancia hospitalaria menor, con una reducción de nueve días, y sin ninguna mortalidad intraoperatoria, a diferencia de la reparación abierta, con un promedio de 18 días de hospitalización y con una mortalidad que alcanzó el 11\% (5 casos) de los pacientes evaluados ${ }^{10}$.

En un estudio realizado por Hollingsworth A, se demostró que, aunque se cumplan criterios para la realización del EVAR, este no se realiza debido a que en el momento en que llega el paciente con la clínica de AAA roto o AAA sintomático, con necesidad quirúrgica, no está disponible el servicio de cirugía vascular, o no se cuenta con el equipo necesario para realizar esta técnica. En este estudio se evaluaron 359 pacientes durante once años, de los cuales 250 tenían AAA roto y 96 AAA sintomático, para evaluar la morfología y hacer la confirmación del diagnóstico 325 pacientes fueron llevados a TC 4 .

De los que presentaron ruptura, 161 fueron sometidos a una reparación abierta, de los cuales 27 necesitaron pinzamiento suprarrenal y 134 requirieron pinza aórtica infrarrenal. En el momento en que estos pacientes llegaron al servicio de urgencias, solo 98 fueron revisados por el equipo de cirugía vascular, mientras que los otros 152 no contaron con esta valoración. De los 96 pacientes 
que presentaron AAA sintomático con requerimiento quirúrgico, 49 fueron valorados por el cirujano vascular y los otros 47 se presentaron cuando no se contaba con este servicio, de estos 39 fueron a reparación abierta y 57 por medio de EVAR. En este artículo no se encontraron datos sobre la diferencia de la mortalidad de los pacientes operados cuando estaba disponible el equipo de cirugía vascular, frente a los intervenidos cuando este no se encontraba en el hospital ${ }^{4}$.

Lamentablemente no existen estudios, que comparen la reparación de AAA roto tratados por cirujanos generales versus cirujanos vasculares, sin embargo los primeros no están capacitados para ofrecer el abordaje EVAR. En países en vías de desarrollo como el nuestro, donde no existe servicio de cirugía vascular en todas las instituciones o no se cuenta con todos los recursos, los cirujanos generales deben estar preparados para poder manejar con abordaje abierto, de forma oportuna y eficaz el AAA roto.

\section{Conclusión}

La reparación mediante el EVAR de un AAA roto es la mejor elección en la actualidad, su uso ha venido en aumento ya que muestra mayores beneficios en comparación con la reparación abierta. Sin embargo, no es la técnica más utilizada como lo podemos ver en nuestro caso, porque se necesita de un personal bien entrenado en cirugía endovascular, además de tener los recursos necesarios para el procedimiento.

Dentro de la formación del cirujano general, se tiene que incluir el aprendizaje de técnicas abiertas, para que puedan abordar a los pacientes que llegan con esta urgencia quirúrgica y de este modo no perder tiempo valioso, para el manejo de esta patología.

\section{Cumplimiento de normas éticas}

Consentimiento informado. Los autores han obtenido el consentimiento informado del paciente referido en el artículo. Este documento obra en poder de los autores. En relación con la confidencialidad de los datos, los autores declaran que han seguido los protocolos de su centro de trabajo sobre la publicación de datos de pacientes.
Conflicto de interés: Ninguno declarado por los autores.

Fuentes de financiación: financiado con recursos propios.

\section{Contribución de los autores:}

Concepción y diseño del estudio: Brandon Steven Aparicio, Marcia Santafe Guerrero, Mauricio Pedraza Ciro, Jean Pulido, Laura Padilla, Luis Felipe Cabrera.

Adquisición de datos: Brandon Steven Aparicio, Marcia Santafe Guerrero, Mauricio Pedraza-Ciro, Jean Pulido, Laura Padilla, Luis Felipe Cabrera.

Análisis e interpretación de datos: Brandon Steven Aparicio, Mauricio Pedraza-Ciro, Luis Felipe Cabrera.

Redacción del manuscrito: Marcia Santafe Guerrero, Mauricio Pedraza-Ciro, Jean Pulido.

Revisión crítica: Brandon Steven Aparicio, Mauricio Pedraza-Ciro, Luis Felipe Cabrera.

\section{Referencias}

1. Takayama T, Yamanouchi D. Aneurysmal disease. The abdominal aorta. Surg Clin North Am. 2013;93:877-91. https://doi.org/10.1016/j.suc.2013.05.005

2. Schanzer A, Messina L. Two decades of endovascular abdominal aortic aneurysm repair: Enormous progress with serious lessons learned. J Am Heart Assoc. 2013;1:1-6. https://doi.org/10.1161/JAHA.111.000075

3. Schermerhorn ML, O’Malley J, Jhaveri A. Endovascular vs. open repair of abdominal aortic aneurysms in the medicare population. NEJM Group. 2008;358:464-74. https://doi.org/10.1056/NEJMoa0707348

4. Hollingsworth AC, Dawkins C, Wong PF, Walker P, Milburn S, Mofidi R. Aneurysm morphology is a more significant predictor of survival than Hardman's index in patients with ruptured or acutely symptomatic abdominal aortic aneurysms. Ann Vasc Surg. 2019:58:222-31. https://doi.org/10.1016/j.avsg.2018.10.018

5. Lahoz C, Gracia CE, Reinares-García L, Bellmunt-Montoya S, Brea HA, Fernández-Heredero A, et al. Recomendaciones de la guía para el diagnóstico y tratamiento del aneurisma de aorta abdominal. Clin Invest Arterioscl. 2015;27:159-65.

https://doi.org/10.1016/j.arteri.2015.01.004

6. Casula E, Lonjedo E, Cerverón MJ, Ruiz A, Gómez J. Revisión de aneurisma de aorta abdominal: hallazgos en la tomografía computarizada multidetector pre y postratamiento. Radiología. 2014:56:16-26. https://doi.org/10.1016/j.rx.2012.11.006

7. López M, Martínez F, Camacho J, Sanabria A, Domínguez LC, Vega V. Incidencia de insuficiencia renal aguda posoperatoria en pacientes con aneurisma de aorta abdominal infrarrenal no roto: comparación entre 
abordaje abierto y endovascular. Rev Colomb Cir. 2015;30:212-19

8. United Kingdom EVAR Trial Investigators, Greenhalgh RM, Brown LC, Powell JT, Thompson SG, Epstein D, et al. Endovascular versus open repair of abdominal aortic aneurysm. N Engl J Med. 2010;362:1863-71. https://doi.org/10.1056/NEJMoa0909305

9. Del Canto-Peruyera P, Alvarez-Salgado A, Calvín-Alvarez P, Botas-Velasco M, Vallina-Victorero Vázquez MJ, et al.
Tratamiento del aneurisma de aorta abdominal roto: ¿EVAR o cirugía abierta? Angiología. 2014;66:300-4. https://doi.org/10.1016/j.angio.2014.05.006

10. Paajanen P, Mäkinen K, Karjalainen J, Saari P, Virkkunen J, Partio T, et al. Effect of endovascular treatment rate on population level outcomes and survival after intact abdominal aortic aneurysm repair. Eur J Vasc Endovasc Surg. 2019;58:698-707.

https://doi.org/10.1016/j.ejvs.2019.04.027 